\title{
Altered expression of Platelet Factor 4 and basic fibroblast growth factor correlates with the inhibition of tumor growth in mice
}

\author{
Peace Mabeta $^{1}$, Michael S. Pepper ${ }^{2}$ \\ Departments of ${ }^{1}$ Physiology and ${ }^{2}$ Immunology, Faculty of Health Sciences, University of \\ Pretoria, Tshwane, South Africa
}

Address for Correspondence:

Dr. Peace Mabeta

P.O. Box 2034

Dept. of Physiology

Faculty of Health Sciences

University of Pretoria

Pretoria 0001

South Africa

Tel: +27 125298354

Fax: +27 125298305

E-mail: peace.mabeta@up.ac.za 


\begin{abstract}
Herein we describe the effects of Taxol on endothelioma cell growth and migration in vitro and on vascular tumor growth in vivo. The effects of Taxol on endothelioma cell growth were determined using the crystal violet assay, while cell migration was measured using the xCELLIgence Real Time Cell Analysis system. To study the effects of Taxol on tumor growth, mice were inoculated with endothelioma cells to induce vascular tumor development and were treated with the drug. At termination, tissue samples from Taxol-treated and control mice were stained with hematoxylin and eosin for histological examination, while blood samples were collected for hematological analysis, as well as for the analysis of the expression of angiogenic markers. In vitro, Taxol inhibited cell growth and migration. The drug also inhibited vascular tumor growth in mice, and this correlated with a recovery of mice from thrombocytopenia. Array analysis of blood samples from mice revealed that there was an increase in the expression of Platelet Factor 4 and a suppression of the proangiogenic molecule basic fibroblast growth factor in Taxol-treated animals. Our findings suggest that Taxol may have potential in the treatment of vascular tumors.
\end{abstract}

Keywords: angiogenesis, vascular tumor, cell migration, Taxol, bFGF, PF4 


\section{Introduction}

Paclitaxel, commonly known as Taxol, is a member of the taxane class of drugs, which are derived from the American Pacific yew tree (Taxus brevifolia). ${ }^{1,2}$ It inhibits microtubule dynamics by binding to the $\mathrm{N}$-terminal region of $\beta$-tubulin, which in turn results in the formation of stable microtubules. ${ }^{3}$ Paclitaxel was the first taxane to enter clinical trials and to receive food and drug association (FDA) approval for cancer treatment. ${ }^{2}$ It is currently used as first line therapy for many common malignancies, including lung, breast, ovarian and head and neck cancers. ${ }^{1,4}$ In addition, it has high antitumor activity against some uncommon malignancies such as the endothelial cell tumors angiosarcoma and Kaposis sarcoma. ${ }^{3}$

Besides its known anticancer properties, another characteristic of paclitaxel, namely, its antiangiogenic activity, started a second life for this compound and has led to the investigation of new therapeutic approaches using the drug. ${ }^{5}$

Dordunoo and colleagues reported the first published evidence about a possible antiangiogenic activity of paclitaxel more than 15 years ago. ${ }^{6}$ Their study showed that Taxol inhibits angiogenesis in the chick chorioallantoic membrane (CAM) model. ${ }^{6}$ Since then, a number of studies have reported on the antiangiogenic effects of Taxol in vitro and in vivo. 7,8 Recent studies further showed that Taxol inhibits the growth and migration of endothelial cells. ${ }^{8}$

Endothelial cell neoplasms such as hemangioendotheliomas and hemangiomas have no definitive treatment. Given the potent direct effects of Taxol on endothelial cells, we sought to investigate the effects of Taxol on the development of vascular tumors in a mouse model previously employed as a model of for these endothelial cell neoplasms. The effects of the drug on endothelioma cell growth and migration, and on the expression of angiogenic markers were also investigated. 


\section{Materials and Methods}

\subsection{Cell culture}

The endothelioma cell line, sEnd.2, derived from Pym $T$ induced vascular tumors in the skin of the thorax of C57BL6 mice was grown in Dulbecco's Minimum Essential Medium (SigmaAldrich, Germany) supplemented with $10 \%$ heat inactivated fetal bovine serum (FBS) (Invitrogen, USA), $20 \mathrm{mM}$ glutamine (Invitrogen, USA), and 1\% penicillin-streptomycin (Whitehead Scientific, South Africa). Bovine microvascular endothelial (BME) cells were maintained in $\alpha$-modified Eagle's Medium, supplemented with $15 \%$ donor calf serum (Invitrogen, USA), and 1\% penicillin-streptomycin (Whitehead Scientific, South Africa). The cell lines were maintained in a $37{ }^{\circ} \mathrm{C}$ incubator in a humidified atmosphere containing $5 \% \mathrm{CO}_{2}$.

\subsection{Cell growth assay}

Cell viability was assessed using the crystal violet nuclear staining assay. Endothelioma cells were seeded in 24-well culture plates at a density of 10000 cells per well for 24 hours, and then treated with Taxol $(0-10 \mu \mathrm{M})$ or dimethylsulfoxide (DMSO) for 48 hours. The time and dose were selected following initial screening over a period of 12 to 72 hours. At termination cells were fixed with $1 \%$ glutaraldehyde in phosphate buffered saline (PBS) for 15 minutes, followed by staining with a $0.1 \%$ crystal violet solution (Sigma-Aldrich, Germany) for 30 minutes. The dye was extracted with $0.1 \%$ Triton X-100 (Sigma-Aldrich, Germany) per well. The absorbance was read at $570 \mathrm{~nm}$ on an ELx 800 Universal Microplate Reader (Bio-Tek instruments Inc., SA). Three wells were analysed for each concentration. Studies were undertaken in triplicate.

\subsection{Cell Migration assay}

Cell migration experiments were performed using 16-well cell invasion and migration (CIM) plates (Roche Applied Science, South Africa). Prior to each experiment, BME and sEnd.2 cells were deprived of FBS for 24 hours. For the migration assay, $160 \mu \mathrm{L}$ serum free medium with or without a chemoattractant, namely, $10 \mathrm{ng} / \mathrm{ml}$ basic fibroblast growth factor (bFGF), as was added to each well of the lower chamber of a CIM plate. The cells were added to the upper chamber of the CIM plate at a density of $6 \times 10^{3}$ cells/well and treated with Taxol $(0-10 \mu \mathrm{M})$ or dimethylsulfoxide (DMSO). The migration of cells through the filter into the bottom chamber was monitored over 20 hours with the xCELLigence RTCA DP instrument (Roche Applied Science, South Africa). The cell index (CI) was recorded by the instrument analyzer and analysis was performed with the supplied RTCA software (vs. 1.2.1). 
Four wells were analysed per condition, and the studies were undertaken in triplicate. Results are based on raw data without CI-normalization.

\subsection{Effects of Taxol on tumor development}

Female C57BL6 mice (8-12 weeks old) were housed at the University of Pretoria Biomedical Research Centre (UPBRC). The mice were given standard commercial food (EPOL) and water ad libitum. Animal experiments were conducted according to a protocol approved by the University of Pretoria Animal Use and Care Committee (project 16/2005).

For tumor induction, $2 \times 10^{6}$ endothelioma cells were inoculated s.c. in the flanks of the mice as previously described. Mice were preloaded with Taxol on day one. The animals were injected with Taxol dose of $6 \mathrm{mg} / \mathrm{kg}$ i.p. Control mice received saline. As no tumor growth was observed 10 days following inoculation in the taxol treated group, a repeat study was undertaken using Taxol doses of 0 (control), 1.2, 3 and $6 \mathrm{mg} / \mathrm{kg}$. The mice were monitored every second day for tumor formation. The mice were sacrificed 15 days after inoculation due to the size of the tumors in the control group, and the tumors were isolated.

\subsection{Histological analysis}

The isolated tumors were fixed in buffered $4 \%$ paraformaldehyde and embedded in paraffin using standard procedures. Five $\mu \mathrm{m}$ sections were cut and stained with hematoxylin and eosin. Sections were viewed under a light microscope and photos were taken using a digital camera.

\subsection{Protein array studies}

The screening of angiogenic proteins in plasma samples of control and Taxol-treated mice was performed using a mouse angiogenic protein array kit (RayBiotech Inc., GA, USA). Membranes spotted in duplicate with antibodies against angiogenic factors were blocked with blocking buffer. This was followed by the incubation of the membranes with biotin-conjugated antibodies against various angiogenic factors (fig. 4). The membranes were subsequently washed with wash buffer and then incubated with horseradish peroxidase-conjugated streptavidin. The intensity of the protein signal (two spots for each protein) was compared with the relative positive signals using the Flourchem Imaging System Software (ProteinSimple, USA). Studies were repeated.

\subsection{Hematological analysis}

For hematological analysis, blood samples were collected in heparinised tubes. The red cell count, hematocrit percentage, haemoglobin concentration, white cell and platelet counts, were determined using a hemocounter, Cell-Dyne 3700 (Abbott, IL, USA). 


\subsection{Data Analysis}

Statistical analyses were performed using either the Student's $t$ test or analysis of variance with the Bonferroni method. Values are expressed as the mean \pm SEM unless stated otherwise. Differences were considered significant when the calculated $p$ value was $<0.05$.

\section{Results}

\subsection{Cell growth}

The effects of Taxol on endothelioma (sEnd.2) cell growth were evaluated over 48 hours. Taxol induced a decrease in sEnd.2 cell numbers in a dose-dependent manner, with an $\mathrm{IC}_{50}$ of about $0.102 \mu \mathrm{M}$ (Fig. 1).

\subsection{Cell Migration}

The migration of host endothelial cells and endothelioma cells appear to contribute to vascular tumor development in the Pym T tumor model. Therefore, the effects of Taxol on cell migration were investigated on a non-transformed endothelial cell line, namely, BME cells and on endothelioma cells. The real-time migration of cells was monitored over 20 hours by measuring electrical impedance using the xCELLigence system. The results showed that Taxol inhibited the migration of both BME and sEnd.2 cells in a dose dependent manner (Fig. 2), with $\mathrm{IC}_{50}$ values of approximately 0.017 and $0.020 \mu \mathrm{M}$ respectively.

\subsection{Tumor development}

All mice in the control group developed tumors at the site of injection on average 6 days after inoculation with sEnd.2 cells (Fig. 3A). Fewer mice developed tumors in groups treated with Taxol (Fig. 3A). However, the tumors that developed in mice treated with 1.2 and $3 \mathrm{mg} / \mathrm{kg}$ of Taxol were smaller in diameter when compared to untreated mice (Fig. 3B). No tumor growth was observed in animals treated with $6.0 \mathrm{mg} / \mathrm{kg}$ Taxol (Fig. 3A,B). Hematoxylin and eosin stained tumor sections from control mice had large blood-filled cavities (Fig. 3C). These hemorrhagic cavities appeared reduced in size in mice treated with Taxol (Fig. 3D).

At lower doses of $1.2 \mathrm{mg} / \mathrm{kg}$, Taxol did not affect the red cell count, haematocrit percentage or haemoglobin concentration (Fig. 3). However, drug doses of $3 \mathrm{mg} / \mathrm{kg}$ and above led to a significant increase in these parameters. 
Although there were fluctuations in the white cell count, there was no significant difference between the values of control and Taxol treated mice (table 1). There was however, a significant dose-dependent increase in the platelet count in mice treated with Taxol (table 1).

Antibody Arrays

The expression of angiogenic proteins was determined in plasma samples. Reduced expression of bFGF and increased expression of Platelet Factor 4 (PF4) were observed in the samples of Taxol-treated mice when compared to control samples (Fig. 4).

\section{Discussion}

Taxol inhibited the growth of endothelioma cells, as well as the migration of non-transformed endothelial cells and endothelioma cells. These observations are consistent with our previously studies which showed that Taxol inhibits the growth of bovine microvascular cells. ${ }^{8}$ Endothelioma cell growth, together with the migration of endothelioma cells and host endothelial cells, have been reported to contribute to the development of Pym T-induced tumors. ${ }^{9}$ Indeed, according to Taraboletti et al. (1993), endothelioma cells secrete a factor that stimulates and directs the migration of host endothelial cells to the site of inoculation. ${ }^{10}$

In vivo, control mice developed tumors at the site of injection following inoculation with sEnd.2 cells. According to Williams et al. (1989), endothelioma cells expressing the middle-T antigen are the primary cause of Pym T-induced tumors, and the continuous presence of these cells sustains the tumor. ${ }^{9}$

The development of the Pym T tumors in mice was associated with a low red cell count, haematocrit, hemoglobin concentration, as well as a decrease in the platelet count. These observations are consistent with the diagnosis of vascular tumors associated with the KasabachMerritt Syndrome (KMS) in human beings. ${ }^{11}$ Following treatment with Taxol, there was recovery of all the affected haematological parameters, including the platelet count. The recovery was dose-dependent, with a dose of $6 \mathrm{mg} / \mathrm{kg}$ being most effective.

A key finding of this study was that Taxol altered the expression of two angiogenic markers, namely PF4 and bFGF. In comparison with control mice, there was an increase in the expression of platelet factor 4 (PF4) in Taxol treated mice. Platelet factor 4, also known as CXCL4 is a 70-amino acid molecule which belongs to the CXC chemokine family. ${ }^{12}$ The factor promotes coagulation, and is a chemoattractant for both neutrophils and fibroblasts. ${ }^{12}$ In addition to its role in coagulation, PF4 appears to have antiangiogenic effects. 
There was a decrease in the expression of bFGF in Taxol-treated mice. This growth factor is a $18 \mathrm{kDa}$ molecule that plays an important during Physiological angiogenesis. ${ }^{13}$ Several lines of research have established that bFGF stimulates angiogenesis under pathological settings too, and the growth factor has also been shown to be increased in various tumors, including vascular tumors in humans. ${ }^{13-16}$

In conclusion, these findings suggest that Taxol might play an important role in inhibiting the growth of these vascular tumors. Further studies are warranted to establish the lowest most effective dose in the treatment of these vascular lesions, and components of the bFGF pathway that could serve as potentially useful therapeutic targets in combination treatment modalities for these vascular tumors.

\section{Disclosure of interest}

The authors declare that there is no conflict of interest to disclose with regard to this article.

\section{Acknowledgements}

This study was supported by the University of Pretoria and the National Research Foundation $(\mathrm{NRF})$. 


\section{References}

1. Rowinsky EK, Donehower RC. Paclitaxel. N Engl J Med 1995; 1004-1014.

2. Guerra-Bubb J, Croteau R, Williams RM. The early stages of Taxol biosynthesis: an interim report on the synthesis and identification of early pathway metabolites. Nat Prod Rep 2012; 29:683-696.

3. Orr AG, Verdier-Pinard P, McDaid H, Horwittz SB. Mechanisms of Taxol resistance related to microtubules. Oncogene 2003; 22:7280-7295.

4. Tran T, Gillet L, Roger S, et al. Non-anti-mitotic concentrations of Taxol reduce breast cancer cell invasiveness. Biochem Biophys Res Commun 2009; 379:304-308.

5. Hayot C, Farinelle S, De Decker R, et al. In vitro pharmacological characterizations of the anti-angiogenic and anti-tumor cell migration properties mediated by microtubule-affecting drugs, with special emphasis on the organization of the actin cytoskeleton. Int J Oncol 2002; 21:417-425.

6. Dordunoo SK, Jackson JK, Arsenault LA, Oktaba AM, Hunter WL, Burt HM. Taxol encapsulation in Poly(Epsilon-Caprolactone) microspheres. Cancer Chemother Pharmacol 1995; 36:279-282.

7. Avramis IA, Kwock R, Avramis VI. Taxotere and vincristine inhibit the secretion of the angiogenesis inducing vascular endothelial growth factor (VEGF) by wild-type and drug-resistant human leukemia T-cell lines. Anticancer Res 2001; 21:2281-2286.

8. Mabeta P, Pepper MS. A comparative study on the anti-angiogenic effects of dna-damaging and cytoskeletal-disrupting agents. Angiogenesis 2009; 12:18-90.

9. Williams RL, Risau W, Zerwes HG, et al. Endothelioma cells expressing the polyoma middle T oncogene induce hemangiomas by host cell recruitment. Cell 1989; 57:1053-1063. 
10. Taraboletti G, Belotti D, Dejana E, Mantovani A, Giavazzi R. Endothelial cell migration and invasiveness are induced by a soluble factor produced by murine endothelioma cells transformed by polyoma virus middle T oncogene. Cancer Res 1993; 53:3812-3816.

11. Sakar M, Mulliken JB, Kozakewich HPW, Robertson RL, Burrows E. Thrombocytopenic coagulopathy is associated with kaposiform hemangioendothelioma and not with common infantile hemangioma. Plast Reconstr Surg 1997; 100:1377-1386.

12. Bikfalvi A. Platelet Factor 4: An inhibitor of angiogenesis. Semin Thromb Hemost 2004; 30:379-385.

13. Gerwins P, Sköldenberg E, Claesson-Welsh L. Function of fibroblast growth factors and vascular endothelial growth factors and their receptors in angiogenesis. Crit Rev Oncol 2000; 34:185-194.

14. Cavallaro U, Soria MR, Montesano R. Exogenous fibroblast growth factor-2 induces a transformed phenotype in vascular kaposis sarcoma-like cells. Mol Cell Biol Res Commun 2000; 4:203-205.

15. Cross MJ CL. FGF and VEGF Function in Angiogenesis: Signalling Pathways, biological responses and therapeutic inhibition. Trends Pharmacol Sci 2001; 22:201-207.

16. Dong X, Han ZC, Yang R. Angiogenesis and antiangiogenic therapy in hematologic malignancies. Crit Rev Oncol 2007; 62:105-118. 


\section{Tables}

Table 1. Blood parameter values in control and Taxol-treated mice. 


\section{Figure Legends}

Figure 1. Taxol inhibits endothelioma cell growth.

Taxol reduced the percentage of viable endothelioma cells in a dose-dependent manner. Data represents mean values \pm SD of three wells from triplicate studies. $* \mathrm{P}<0.05$ compared to control.

Figure 2. Effects of Taxol on cell migration.

Taxol induced a decrease in the migration of both BME and sEnd. 2 cells.

Figure 3. Taxol inhibits vascular tumor growth in mice.

The percentage of mice that development tumors (tumor incidence) (A) and tumor size (B) were reduced inTaxol treated mice compared to control mice. $\mathrm{n}=5$ mice per group, $* \mathrm{P}<0.05$

Representative histology images of tissue samples revealed vascular tumors with a large bloodfilled lumens (C-D); however the hemorrhagic lumen was reduced following treatment with 3 $\mathrm{mg} / \mathrm{kg}$ Taxol (D). Bar $=20 \mu \mathrm{m}$.

Figure 4. Analysis of Protein expression

At termination, blood samples were collected from control and Taxol-treated mice and protein array analysis was undertaken. 


\begin{tabular}{|c|c|c|c|c|c|}
\hline & & & & Taxol & $(m g / k g)$ \\
\hline & Baseline & Control & 1.2 & 3 & 6 \\
\hline $\begin{array}{l}\text { RCC } \\
\left(10^{6} / \mu \mathrm{L}\right)\end{array}$ & $12.82 \pm 0.46$ & $4.70 \pm 2.78^{*}$ & $4.89 \pm 2.29^{*}$ & $8.80 \pm 0.55^{\star \star}$ & $8.50 \pm 0.47^{\star *}$ \\
\hline $\begin{array}{l}\mathrm{Hb} \\
(\mathrm{g} / \mathrm{dL})\end{array}$ & $138.22 \pm 2.57$ & $75.54 \pm 9.27^{*}$ & $79.46 \pm 7.37^{*}$ & $135.12 \pm 2.04^{\star *}$ & $137.00 \pm 4.36^{\star \star}$ \\
\hline $\mathrm{HR}(\%)$ & $40.86 \pm 2.63$ & $23.06 \pm 3.5^{\star}$ & $25.16 \pm 11.7^{*}$ & $41.35 \pm 2.65^{\star \star}$ & $40.60 \pm 5.05^{\star \star}$ \\
\hline $\begin{array}{l}\text { THRC } \\
\left(10^{3} / \mu \mathrm{L}\right)\end{array}$ & $704.01 \pm 0.58$ & $67.36 \pm 0.65^{\star}$ & $224.01 \pm 0.25^{\star}$ & $603.75 \pm 01.08^{\star *}$ & $665.67 \pm 0.62^{* *}$ \\
\hline $\begin{array}{l}\text { WCC } \\
\left(10^{3} / \mu L\right)\end{array}$ & $6.05 \pm 0.25$ & $4.98 \pm 2.45$ & $4.51 \pm 1.67$ & $4.83 \pm 0.28$ & $4.61 \pm 0.56$ \\
\hline
\end{tabular}

Values are shown as mean \pm SEM. $\mathrm{n}=5$; $* \mathrm{P}<0.05$ compared to baseline (healthy mice not inoculated with endothelioma cells; $* * \mathrm{P}<0.05$ compared to control) 


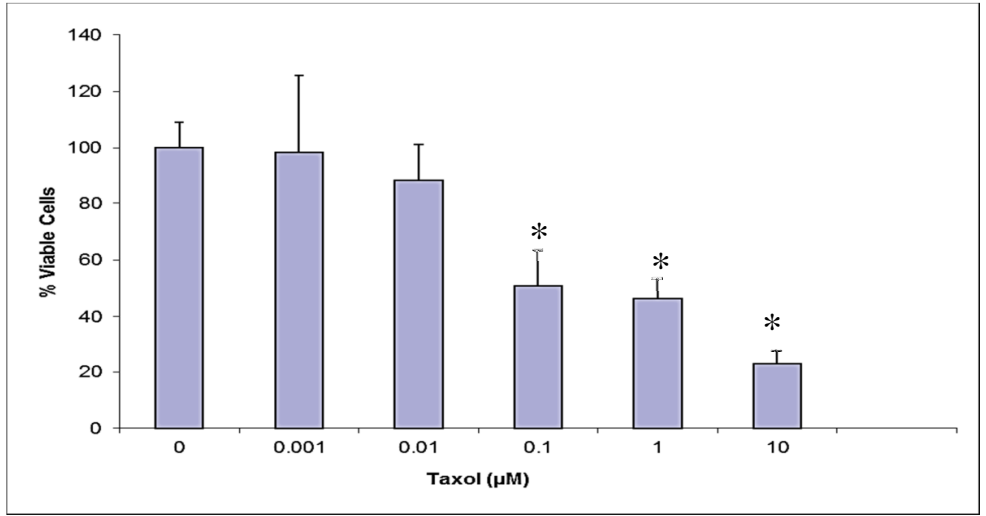




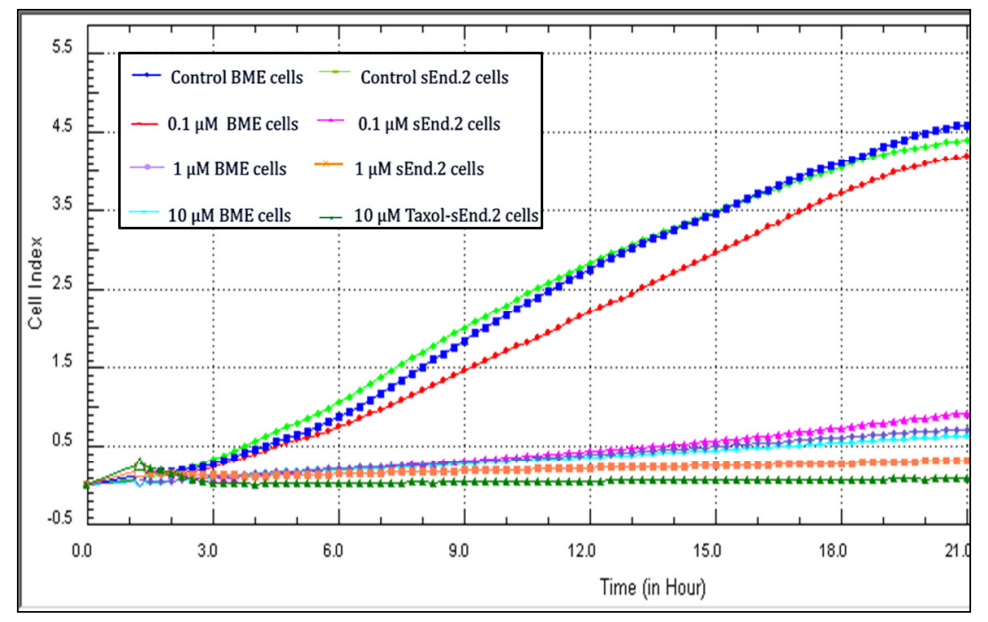



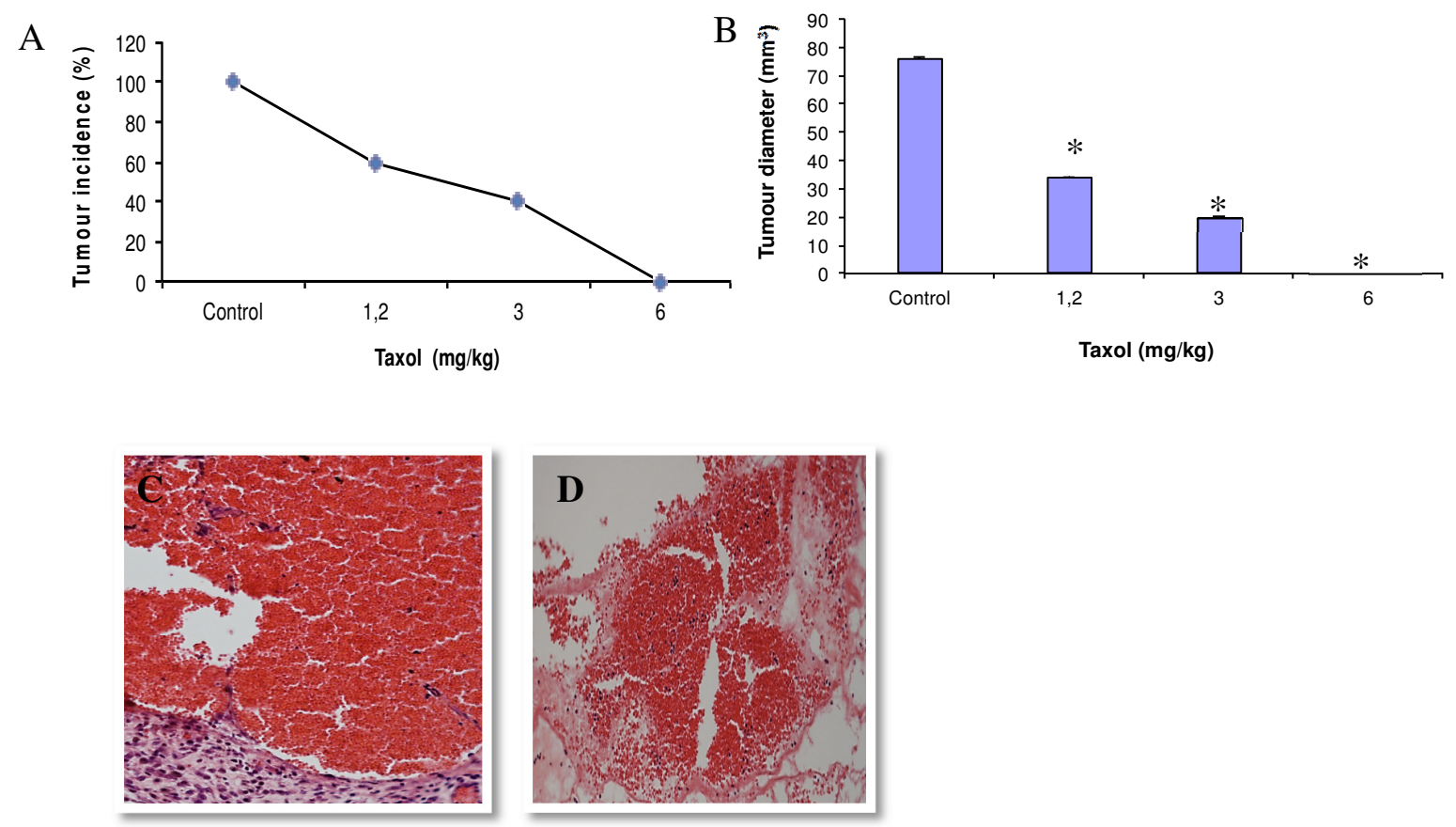


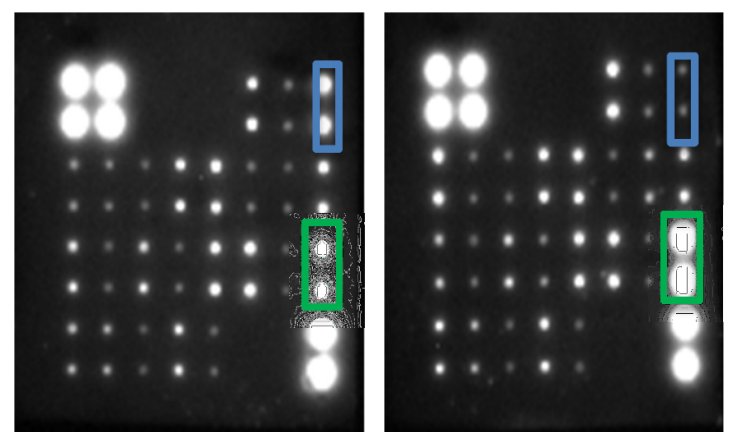

\begin{tabular}{|c|c|c|c|c|c|c|c|c|}
\hline & A & B & C & D & E & F & G & H \\
\cline { 2 - 9 } & POS & POS & NEG & NEG & BLANK & Eotaxin-1 & Fas Ligand & bFGF \\
\hline 2 & POS & POS & NEG & NEG & BLANK & Eotaxin-1 & Fas Ligand & bFGF \\
\hline 3 & G-CSF & GM-CSF & $\begin{array}{c}\text { IFN } \\
\text { gamma }\end{array}$ & IGF-2 & IL-1 alpha & IL-1 beta & IL-12p40/70 & IL-12p70 \\
\hline 4 & G-CSF & GM-CSF & $\begin{array}{c}\text { IFN } \\
\text { gamma }\end{array}$ & IGF-2 & IL-1 alpha & IL-1 beta & IL-12p40/70 & IL-12p70 \\
\hline 5 & IL-13 & IL-6 & IL-9 & Leptin & MCP-1 & M-CSF & MIG & PF-4 \\
\hline 6 & IL-13 & IL-6 & IL-9 & Leptin & MCP-1 & M-CSF & MIG & PF-4 \\
\hline 7 & TIMP-1 & TIMP-2 & TNF-alpha & THPO & VEGF & BLANK & BLANK & POS \\
\hline 8 & TIMP-1 & TIMP-2 & TNF-alpha & THPO & VEGF & BLANK & BLANK & POS \\
\hline
\end{tabular}

POS = Positive control spot NEG = Negative control spot BLANK = Blank spot 